\title{
A multi-purpose watermarking scheme based on hybrid of lifting wavelet transform and Arnold transform
}

\begin{abstract}
This paper introduces a new multi-purpose image watermarking algorithm which based on a hybrid of lifting wavelet transform (LWT) and Arnold transform for copyright protection and image authentication. In the proposed scheme, the original image is first decomposed by LWT into four subbands. Then the robust watermark which is a binary logo image is decomposed by Discrete Wavelet Transform (DWT) as such only the high frequency subband of the watermark are embedded in the low frequency subband of the original image. The fragile watermark is block wise self-generated from the original image and scrambled using Arnold transform which is later embedded in the spatial domain of the robust watermarked image. Self-generated fragile watermark supports self-authentication with high localization, whereas scrambling the fragile watermark increases the security of the algorithm. On the other hand, the lifting scheme approaches have almost one half the amounts of operations compared to the DWT based approaches. The overall system has been tested against various attacks and the results demonstrated that the robust watermark can be decoded successively under each attack. In addition, the proposed algorithm can detect any tampering attempts.
\end{abstract}

Keyword: Fragile watermark; Multipurpose watermarking system; Robust watermark 